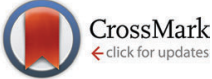

Cite this: Phys. Chem. Chem. Phys., 2015, 17, 32289

Received 25th September 2015, Accepted 7th November 2015

DOI: $10.1039 / c 5 c p 05749 c$

\section{Probing of chain conformations in conjugated polymer nanoparticles by electron spin resonance spectroscopy $\dagger$}

\author{
C. Hintze, $\ddagger$ F. Schütze, $\ddagger$ M. Drescher* and S. Mecking*
}

\begin{abstract}
In the case of conjugated polymer chains usually considered as rigid or stiff, it is an open question how the individual chains adopt their conformation inside nanoparticles. Here, the conformation of such a rigid conjugated polymer chain is elucidated for the first time. For this purpose, electron paramagnetic resonance spectroscopy as a method allowing for a direct observation is established.
\end{abstract}

www.rsc.org/pccp

\section{Introduction}

Conjugated polymers possess favourable properties like conductivity, photo- and electroluminescence ${ }^{1}$ and light-induced charge generation useful for a variety of applications, increasingly including nanoparticles. ${ }^{2-12}$ Particle sizes as low as $8 \mathrm{~nm}$ have been reported frequently. ${ }^{13}$

This raises the intriguing question of the polymer chain conformation in such particles. Conjugated polymers are generally considered to possess a rigid chain nature. For poly(phenylene ethynylene)s in solution a persistence length of $14 \mathrm{~nm}$ at room temperature is found. ${ }^{14}$ This appears contradictive to the existence of smaller size nanoparticles, and it remains unclear how conjugated polymer chains actually pack into such nanoparticles.

To access chain conformations experimentally, a determination of distance distributions (of chain ends) is required. FRET as a fluorescence based method is of limited utility for studies of strongly fluorescent particles as these will interfere with the observation of the labels. Here, we utilize dipole-dipole coupling to determine inter-spin distances by double-electron-electron-resonance (DEER) (also referred to as PELDOR). This pulsed electron spin resonance (ESR) technique has been employed to obtain structural information on a 1.5-10 nm length scale in biomacromolecules, ${ }^{15-23}$ and for probing the conformation of rod-like molecules in solution. ${ }^{14,24-27}$ The commonly used nitroxides ${ }^{28}$ as spin labels ${ }^{29,30}$ are also smaller than fluorophores, and thus interfere less with the system of interest. ${ }^{31,32}$

Department of Chemistry, University of Konstanz, 78464 Konstanz, Germany. E-mail: Malte.Drescher@uni-konstanz.de, Stefan.Mecking@uni-konstanz.de

$\dagger$ Electronic supplementary information (ESI) available: Material characterization, details on bending energy $v s$. surface energy, sample preparation for ESR, and data analysis procedures. See DOI: $10.1039 / \mathrm{c} 5 \mathrm{cp} 05749 \mathrm{c}$

$\$$ These authors contributed equally.
On a molecular basis, $\pi$-conjugated oligomers are referred to as rigid rods, ${ }^{33}$ which makes them desirable as building blocks for (supra-)molecular architectures. Oligomeric phenylene ethynylenes $\left(\mathrm{OPEs}^{34}\right)$ have been intensively studied e.g. as parts of nanomachines, ${ }^{35,36}$ for the construction of shape-persistent macrocycles $^{37,38}$ and as spacers between electronically interacting moieties. ${ }^{39-41}$ The intrinsic flexibility of the OPEs ${ }^{26,42}$ has been studied, too. ${ }^{14,27}$

\section{Experimental}

\section{Synthesis}

General oligomer synthesis. The synthesis of monodisperse HO-OPE $_{n}$-OH $(n=5,7,9,11,21)$, HO-OPE ${ }_{4}$, HO-OPE $_{22}$ and PEGOPE $_{\mathbf{9}}$ has been reported previously. ${ }^{43}$

DCC-coupling - general procedure for the preparation of TEMPO labeled DL-OPE $\boldsymbol{n}_{\boldsymbol{n}}$ oligomers. 2-3 Eq. 4-carboxy-2,2,6,6-tetramethylpiperidine-1-oxyl, 0.4 eq. 4-(dimethylamino)pyridine and 3 eq. dicyclohexylcarbodiimide were dissolved in dry dichloromethane at $0{ }^{\circ} \mathrm{C}$. The reactants were stirred for $10 \mathrm{~min}$, followed by addition of $\mathbf{H O}-\mathbf{O P E}_{\boldsymbol{n}}-\mathbf{O H}$. The reaction was stirred for 1-3 days until ${ }^{1} \mathrm{H}$-NMR spectroscopy confirmed complete conversion of the alcohol. A colorless precipitate was filtered off over a syringe filter. The solvent was removed yielding the crude product. Further product purification are described in detail in the respective procedures for the individual components as follows.

SL-OPE $\mathbf{4}_{4}$. See also general procedure for DCC-coupling. HO$\mathbf{O P E}_{4}(50 \mathrm{mg}, 34 \mu \mathrm{mol})$, 4-carboxy-2,2,6,6-tetramethylpiperidine1-oxyl (10 mg, $50 \mu \mathrm{mol}), 4$-(dimethylamino)pyridine $(1.6 \mathrm{mg}$, $13 \mu \mathrm{mol})$ and dicyclohexylcarbodiimide $(14 \mathrm{mg}, 67 \mu \mathrm{mol})$ were stirred for $18 \mathrm{~h}$ in $2 \mathrm{~mL}$ of dichloromethane. The white precipitate was removed by filtration over a syringe filter. The solvent was removed and the residue was purified by column 
chromatography (pentane/EE $6: 1$ ). The product was obtained as a yellow oil (51 mg, 90\%).

DL-OPE ${ }_{5}$. See also general procedure for DCC-coupling. HO$\mathbf{O P E}_{5}-\mathbf{O H}(90 \mathrm{mg}, 50 \mu \mathrm{mol}), 4$-carboxy-2,2,6,6-tetramethylpiperidine1-oxyl (30 mg, $150 \mu \mathrm{mol}), 4$-(dimethylamino)pyridine $(2.4 \mathrm{mg}$, $15 \mu \mathrm{mol})$ and dicyclohexylcarbodiimide ( $41 \mathrm{mg}, 200 \mu \mathrm{mol})$ were stirred for 3 days in $3 \mathrm{~mL}$ of dichloromethane. The white precipitate was removed by filtration over a syringe filter. The solvent was removed and the residue was purified by column chromatography (pentane/EE $4: 1$ ). The product was obtained as a yellow solid ( $80 \mathrm{mg}, 72 \%$ ).

DL-OPE $_{7}$. See also general procedure for DCC-coupling. HOOPE $_{7}-\mathbf{O H}(60 \mathrm{mg}, 23$ mol), 4-carboxy-2,2,6,6-tetramethylpiperidine1-oxyl (14 mg, $70 \mu \mathrm{mol})$, 4-(dimethylamino)pyridine (1.1 mg, $9 \mu \mathrm{mol})$ and dicyclohexylcarbodiimide ( $19 \mathrm{mg}, 93 \mu \mathrm{mol}$ ) were stirred for $18 \mathrm{~h}$ in $3 \mathrm{~mL}$ of dichloromethane. The white precipitate was removed by filtration over a syringe filter. The solvent was removed and the residue was purified by column chromatography (pentane/EE 6:1). The product was obtained as a yellow solid (51 mg, 90\%).

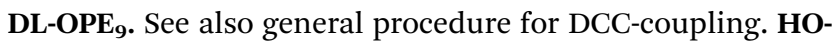

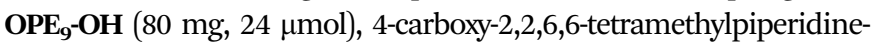
1-oxyl (15 mg, $75 \mu \mathrm{mol}), 4$-(dimethylamino)pyridine $(1.2 \mathrm{mg}$, $10 \mu \mathrm{mol})$ and dicyclohexylcarbodiimide $(20 \mathrm{mg}$, $97 \mu \mathrm{mol})$ were stirred for 2 days in $3 \mathrm{~mL}$ of dichloromethane. The oligomer was precipitated in methanol. Column chromatography (pentane/EE $5: 1$ ) gave the product as a yellow solid (72 $\mathrm{mg}, 82 \%)$.

DL-OPE $_{11}$. See also general procedure for DCC-coupling. HO$\mathbf{O P E}_{11}-\mathbf{O H}(60 \mathrm{mg}, 15 \mu \mathrm{mol})$, 4-carboxy-2,2,6,6-tetramethylpiperidine1-oxyl (9 mg, $45 \mu \mathrm{mol})$, 4-(dimethylamino)pyridine $(0.7 \mathrm{mg}, 6 \mu \mathrm{mol})$ and dicyclohexylcarbodiimide $(12 \mathrm{mg}, 60 \mu \mathrm{mol})$ were stirred for $18 \mathrm{~h}$ in $3 \mathrm{~mL}$ of dichloromethane. The oligomer was precipitated in methanol. Column chromatography (pentane/EE 5:1) gave the product as a yellow solid (49 $\mathrm{mg}, 75 \%$ ).

DL-OPE $_{21}$. See also general procedure for DCC-coupling. HO$\mathbf{O P E}_{21}$-OH (30 mg, $\left.4 \mu \mathrm{mol}\right)$, 4-carboxy-2,2,6,6-tetramethylpiperidine1-oxyl (2.4 mg, $12 \mu \mathrm{mol}), 4$-(dimethylamino)pyridine $(0.2 \mathrm{mg}$, $1.5 \mu \mathrm{mol})$ and dicyclohexylcarbodiimide $(3.2 \mathrm{mg}, 16 \mu \mathrm{mol})$ were stirred for 2 days in $3 \mathrm{~mL}$ of dichloromethane. The oligomer was precipitated in methanol and gave the product as a yellow solid (25 $\mathrm{mg}, 83 \%$ ).

SL-OPE $\mathbf{2 2}_{22}$. See also general procedure for DCC-coupling. HO$\mathbf{O P E}_{22}$ (30 mg, 4 $\mu \mathrm{mol}$ ), 4-carboxy-2,2,6,6-tetramethylpiperidine1-oxyl (2.3 mg, $11 \mu \mathrm{mol}), 4$-(dimethylamino)pyridine $(0.2 \mathrm{mg}$, $1.5 \mu \mathrm{mol})$ and dicyclohexylcarbodiimide $(3 \mathrm{mg}, 15 \mu \mathrm{mol})$ were stirred for 2 days in $2 \mathrm{~mL}$ of dichloromethane. A white precipitate was removed by filtration over a syringe filter. The oligomer was precipitated in methanol and gave the product as a yellow solid (21 mg, 65\%).

\section{ESR-samples}

(A) DL-OPE 5 (9 $\mu \mathrm{g}, 4 \mathrm{nmol})$ was mixed with PEG-OPE 9 (4.1 $\mathrm{mg}$, $560 \mathrm{nmol})$ and $\mathbf{H O}-\mathbf{O P E}_{21}-\mathbf{O H}(4.2 \mathrm{mg}, 550 \mathrm{nmol})$ in $4 \mathrm{~g}$ of tetrahydrofuran. The solution was injected rapidly into $40 \mathrm{~mL}$ of Milli-Q water and the dispersion was stirred for $15 \mathrm{~min}$. The organic solvent was removed and the dispersion was freezedried.
(B) (a) DL-OPE $21(200 \mu \mathrm{g}, 26 \mathrm{nmol})$ was mixed with PEG-OPE $\mathbf{~}_{\mathbf{9}}$ $(4.2 \mathrm{mg}, 570 \mathrm{nmol})$ and $\mathbf{H O}-\mathbf{O P E} \mathbf{E}_{\mathbf{2 1}} \mathbf{- O H}(3.7 \mathrm{mg}, 490 \mathrm{nmol})$ in $3 \mathrm{~g}$ of tetrahydrofuran. The solution was injected rapidly into $30 \mathrm{~mL}$ of Milli-Q water and the dispersion was stirred for $15 \mathrm{~min}$. The organic solvent was removed and the dispersion was freeze-dried. (B) (b) DLOPE $_{21}(48 \mu \mathrm{g}, 7 \mathrm{nmol}$ ) was mixed with PEG-OPE 9 (7.1 mg, $970 \mathrm{nmol}$ ) in $3.5 \mathrm{~g}$ of tetrahydrofuran. The solution was injected rapidly into $35 \mathrm{~mL}$ of Milli-Q water and the dispersion was stirred for $15 \mathrm{~min}$. The organic solvent was removed and the dispersion was freezedried. (C) SL-OPE $22(820 \mu \mathrm{g}, 100 \mathrm{nmol})$ was mixed with PEG-OPE $(4.1 \mathrm{mg}, 560 \mathrm{nmol})$ and $\mathbf{H O}-\mathbf{O P E}_{21}-\mathbf{O H}(4.1 \mathrm{mg}, 540 \mathrm{nmol})$ in $4 \mathrm{~g}$ of tetrahydrofuran. The solution was injected rapidly into $40 \mathrm{~mL}$ of Milli-Q water and the dispersion was stirred for $15 \mathrm{~min}$. The organic solvent was removed and the dispersion was freeze-dried.

\section{ESR experiments}

DEER-experiment. The DEER experiment was performed in Q-band using an Elexsys E580 spectrometer (Bruker Biospin) equipped with a 15 watt solid state microwave amplifier and a helium gas flow system (CF935, Oxford Instruments). The fourpulse, dead-time free DEER sequence is given by: $\pi / 2_{\mathrm{obs}}-\tau_{1}-$ $\pi_{\mathrm{obs}}-t-\pi_{\text {pump }}-\left(\tau_{1}+\tau_{2}-t\right)-\pi_{\mathrm{obs}}-\tau_{2}-$ echo. The echo amplitude is observed as a function of time $t$ starting with $t=280 \mathrm{~ns}$. The pump pulse (typically $34 \mathrm{~ns}$ corresponding to a $\pi$-pulse) was set to the maximum of the nitroxide spectrum and the observer pulse was set $40 \mathrm{MHz}$ higher; $\pi / 2$ and $\pi$ pulses at observer frequency were of typically $30 \mathrm{~ns}$ and $60 \mathrm{~ns}$ length, respectively. The probes in solution were measured at optimized values for $\tau_{2}$ to cover more than one full modulation. In this case, nuclear modulation averaging was utilized to avoid artifacts from the deuterium nuclear modulation. The probes in particles were measured at maximum values for $\tau_{2}$ yielding sufficient signalto-noise ratio without nuclear modulation averaging. The accumulation time was typically 18 hours at $50 \mathrm{~K}$.

Cw-ESR measurements. Continuous wave (cw-) ESR spectra were recorded at $120 \mathrm{~K}$ on an Elexsys E580 spectrometer (Bruker Biospin) equipped with a helium gas flow system (CF935, Oxford Instruments). Spectra were obtained with s sweep width of $300 \mathrm{G}$, a modulation amplitude of $3000 \mathrm{mG}$, a modulation frequency of $100 \mathrm{kHz}$, and a microwave power of $0.2 \mathrm{~mW}$. The signal-to-noise ratio was improved by accumulation of 5 spectra featuring $42 \mathrm{~s}$ scan time each. Signal intensities were determined via the double integral of the first derivative ESR spectrum. The spectra were corrected for frequency differences for visualization.

\section{Results and discussion}

We chose OPEs as a system to study the arrangement of conjugated chains in nanoparticles. Strictly monodisperse defect free $\mathbf{O P E}_{\boldsymbol{n}}$ $(n=4,5,7,9,11,21,22)$, PEG-OPE ${ }_{9}\left(M_{\mathrm{n}(\mathrm{PEG})}=2000 \mathrm{~g} \mathrm{~mol}^{-1}\right)$, and PEG-OPE $21\left(M_{\mathrm{n}(\mathrm{PEG})}=5000 \mathrm{~g} \mathrm{~mol}^{-1}\right)$ were synthesized. ${ }^{43}$ Spin labeling was conducted via DCC coupling of the dihydroxy endgroup functionalized $\mathbf{O P E}_{\boldsymbol{n}}$ with 4-Carboxy-2,2,6,6-tetramethylpiperidine-1oxyl, yielding the singly and doubly TEMPO-labeled oligomers, SL-OPE $_{n}$ and DL-OPE ${ }_{n}$, respectively (ESI $\dagger$ ). 


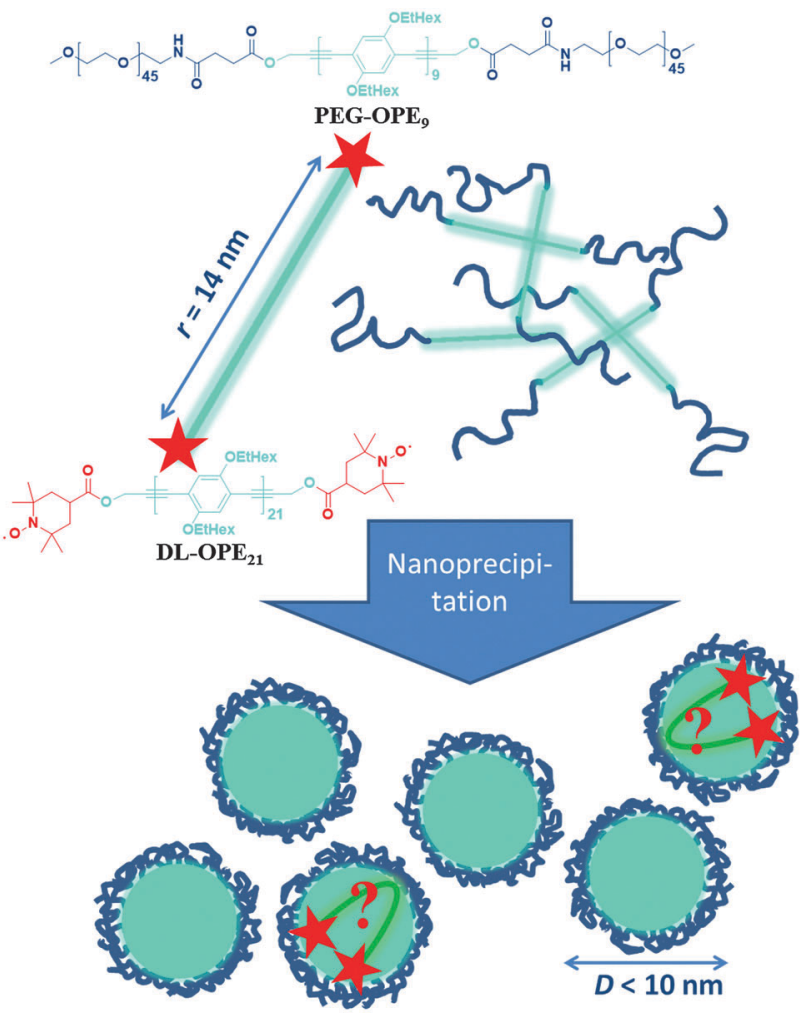

Scheme 1 Schematic representation of particle formation from amphiphilic block copolymer PEG-OPE 9 and DL-OPE P1 $_{1}$

Aqueous dispersions from the obtained PEG-OPE $\boldsymbol{n}_{\boldsymbol{n}}$ block copolymers were generated via nanoprecipitation, ${ }^{44-46}$ a common technique for the generation of conjugated polymer nanoparticles. ${ }^{47}$ A dilute tetrahydrofuran solution was injected into an excess of water (Scheme 1). The polymer forms nanoparticles, self-stabilized by the hydrophilic PEG. Such amphiphilic block copolymers can be used for the incorporation of other hydrophobic molecules. ${ }^{48}$ Coprecipitation of PEG-OPE $9\left(M_{\mathrm{n}(\mathrm{PEG})}=2000 \mathrm{~g} \mathrm{~mol}^{-1}\right)$ block copolymer and $\mathbf{O P E}_{21}$ homopolymer (contour length $L_{21} \approx$ $14 \mathrm{~nm}$, Fig. 2) in a one to one ratio, leads to the formation of small sized particles with an average particle size of $D_{\text {small }} \approx$ $8 \pm 2 \mathrm{~nm}$ (determined by dynamic light scattering, Fig. 1). Without the addition of $\mathbf{O P E}_{21}$, larger particles of about $D_{\text {big }} \approx$ $14 \pm 1 \mathrm{~nm}$ are formed. With addition of $\mathbf{O P E}_{21}$, the existence of
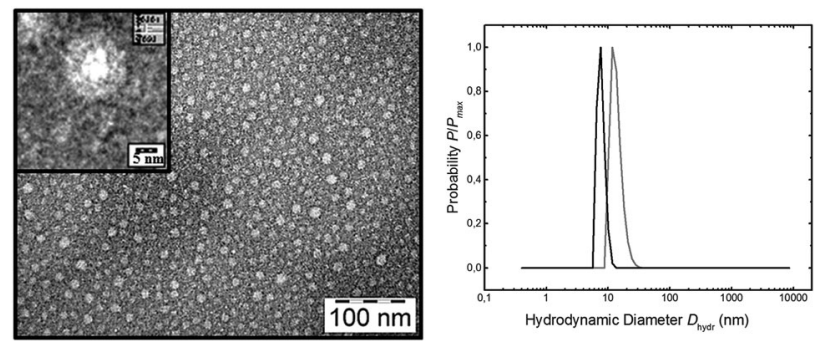

Fig. 1 Left: TEM image of nanoparticles from PEG-OPE $/ \mathrm{OPE}_{\mathbf{2 1}}$ mixtures (stained with phosphotungstic acid), right: number average particle size distribution, obtained by DLS of the obtained nanoparticles from PEG$\mathrm{OPE}_{\mathbf{9}} / \mathrm{OPE}_{21}$ mixtures (black) and from PEG-OPE (grey). small size particles has been verified by TEM (Fig. 1), yet the difference in size between the particles from pure $\mathbf{P E G - O P E _ { n }}$ and PEG-OPE ${ }_{n} / \mathbf{O P E}_{21}$ mixtures is difficult to quantify by transmission electron microscopy since the soft particles flatten out on the TEM grid.

These observations raise an intriguing question: If $\mathbf{O P E}_{\mathbf{2 1}}$ facilitates small particle formation, what is its conformation inside these particles given that its chain length is almost twice the particle diameter. This issue was unraveled by ESR distance measurements in the solid particles.

For the preparation of nanoparticles for ESR studies, in the above procedure a small amount of $\mathbf{O P E}_{21}$ was replaced by DL-OPE $\mathbf{2}_{21}$, with the concentration of the latter being so low, that on average only one labeled chain per particle can be assumed, which corresponds to a spin concentration of $1.6 \mu \mathrm{M}$. The organic solvent was removed and the dispersion was freeze-dried.
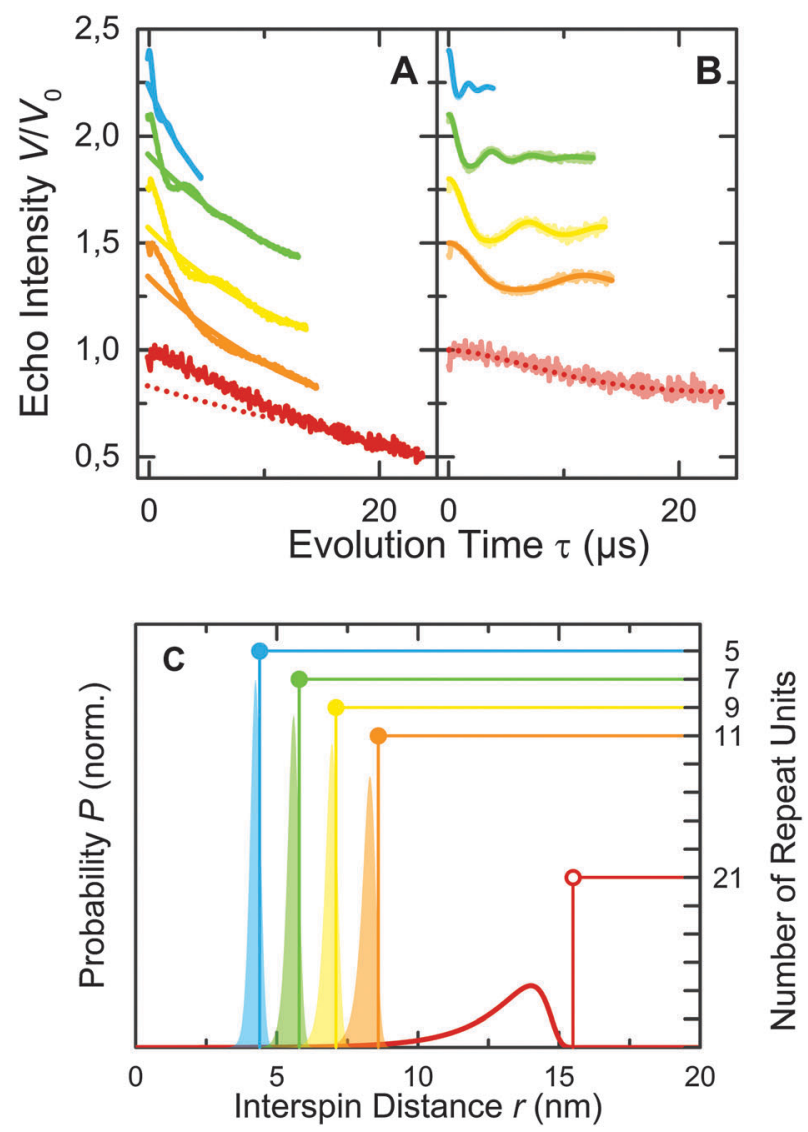

Fig. 2 DEER measurements of DL-OPE $n$ with $n=5,7,9,11$, and 21 (from blue to red) in deuterated toluene, recorded at $50 \mathrm{~K}$ in Q-band. (A) Normalized DEER traces with intermolecular background contribution. (B) Background corrected DEER form factors with fits of a worm like chain model including Gaussian broadening. (C) Filled curves represent distance distributions $P(r)$ obtained by fits to the DEER form factor. The curve for $\mathrm{DL}_{-} \mathrm{OPE}_{\mathbf{2 1}}$ is not a fit but extrapolated. The contour length of DL-OPE $\boldsymbol{n}_{\boldsymbol{n}}$ can be described with a linear function in $n: L_{n^{\prime}}=(0.69 n+0.97) \mathrm{nm}$. The experimental (extrapolated) contour lengths $L_{n^{\prime}}$ are represented by filled (open) dots. The dotted DEER form factor and background in A for $\mathbf{O P E}_{\mathbf{2 1}}$ is calculated from the expected distance distribution in $B$ with an approximate modulation depth and an approximate background density. 
Table 1 Parameters of WLC interspin distance distributions $P(r)$ of DL$\mathrm{OPE}_{n}$ in various environments

\begin{tabular}{|c|c|c|c|c|}
\hline Probe DL- & Environment & $L_{n}{ }^{a}[\mathrm{~nm}]$ & $L_{\mathrm{p}}^{b}[\mathrm{~nm}]$ & $\sigma^{c}[\mathrm{~nm}]$ \\
\hline $\mathrm{OPE}_{5}$ & Toluene- $d_{8}$-solution & 4.4 & 18 & 0.22 \\
\hline $\mathrm{OPE}_{7}$ & Toluene- $d_{8}$-solution & 5.8 & 22 & 0.22 \\
\hline $\mathrm{OPE}_{9}$ & Toluene- $d_{8}$-solution & 7.1 & 27 & 0.22 \\
\hline $\mathrm{OPE}_{11}$ & Toluene- $d_{8}$-solution & 8.6 & 30 & 0.22 \\
\hline $\mathrm{OPE}_{21}$ & $o$-Terphenyl- $d_{14}$-solution & 15.5 & 15 & 0.22 \\
\hline $\mathrm{OPE}_{5}$ & PEG-OPE $_{9}$-a particles & 4.5 & 9 & $4 \times 10^{-3}$ \\
\hline
\end{tabular}

We performed DEER measurements on a range of DL-OPE $\boldsymbol{n}_{\boldsymbol{n}}$, with $n=5,7,9$, and 11, in a glassy solution of deuterated toluene at $50 \mathrm{~K}$ (Fig. 2) in order to characterize the OPEs used when not confined in nanoparticles. The background corrected DEER form factor was fitted with a worm like chain model with Gaussian broadening, accounting for the label flexibility (cf. Table 1 for all fit parameters). ${ }^{49}$ Due to limitations of the WLC model for contour lengths $L_{n}$ around the persistence length $L_{\mathrm{p}}$, there is a shift in fitted persistence length with contour length. ${ }^{14}$ The maximum evolution time achieved is not sufficient to fit a distance distribution in the case of DL-OPE $\mathbf{2 1}_{\mathbf{2 1}}$. From the distance distributions of the other oligomers, we are able to extrapolate the distance distribution for $\mathbf{D L}-\mathbf{O P E} \mathbf{2 1}_{\mathbf{2 1}}$. This is important because this is the very OPE that facilitates formation of small nanoparticles as outlined (also $c f$. Table 1).

We investigated the behavior of $\mathbf{D L}^{-\mathbf{O P E}_{5}}$ in particles compared to their behavior in solution. The particles were prepared

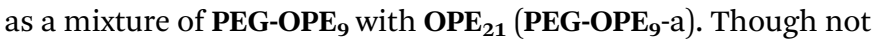
of primary interest, $\mathbf{D L}^{-\mathbf{O P E}_{\mathbf{5}}}$ was chosen as an appropriate test candidate for our method because it is the shortest one of the probes used in this work.

In order to eliminate intermolecular spin-spin interactions, i.e. for the statistically possible case of more than one labeled molecule per particle, we performed control experiments exploiting singly labeled molecules (see ESI $\dagger$ for a detailed discussion).

Upon correction for intermolecular contributions, the data yields distance distributions for both conditions, in solution as well as in particles (Fig. 3). There is a significant loss in echo intensity due to faster electron spin transverse relaxation rate in protonated particles compared to deuterated solvents. With the evolution time achieved, distance measurements on other DL-OPE $_{n}$ (for $n=7,9,11$ ) were not taken into account since their contour length is beyond the distance range accessible with this evolution time. Nevertheless, DEER data can be acquired with sufficient quality in terms of evolution time and signal to noise for distances up to around $5 \mathrm{~nm}$ in nanoparticles. The experiment also shows, that modulation depths normalized to pump pulse lengths in particle samples are around $9 \%$ for quantitative incorporation of the spin labeled probe. This is due to reduction of spin labels during the particle preparation process (see ESI $\dagger$ ).

The distance distribution of $\mathbf{D L}-\mathbf{O P E}_{\mathbf{5}}$ inside the particles reflects a shorter persistence length $L_{\mathrm{p}}$ than in the glassy state

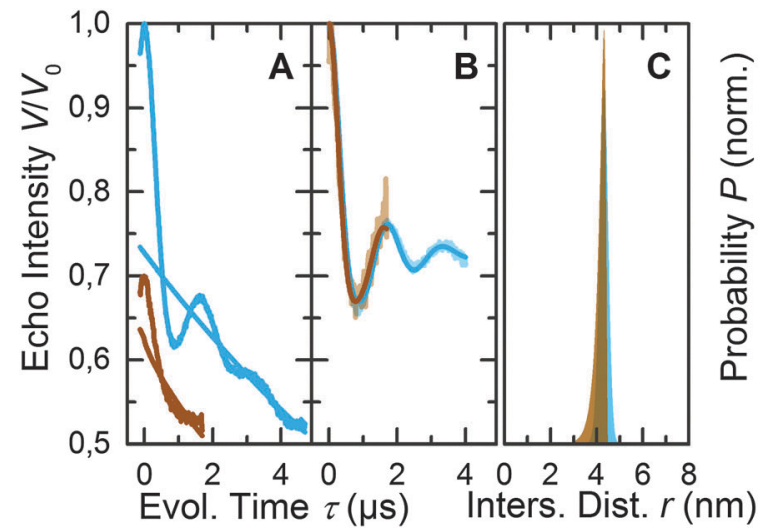

Fig. 3 DEER distance measurements of ${\mathrm{DL}-O P E_{5}}_{\mathbf{5}}$ in deuterated toluene (blue) and in particles (brown) at $50 \mathrm{~K}$ in Q-band. (A) Normalized DEER traces with intermolecular background contribution. (B) Background corrected DEER form factors with fits of a worm like chain model including Gaussian broadening. The DEER form factor of DL-OPE $\mathbf{5}_{\mathbf{5}}$ in particles is vertically stretched by a factor of 3.4 for comparison. (C) Resulting distance distributions $P(r)$

of toluene (Table 1). Since the persistence length depends on the temperature, and considering that the particle preparation was performed at room temperature, which is significantly higher than the glass transition temperature of toluene, this is as expected. Furthermore, the standard deviation of the additional Gaussian broadening due to linker flexibility converges to zero in the fits. This can be explained by the more restricting environment surrounding the label in the particles compared to the situation in solution. Despite both effects have to be taken with caution due to the limited evolution time, Fig. 3 clearly shows that the obtained distance distribution does not change significantly.

While the contour length of DL-OPE $\mathbf{5}_{5}$ is smaller than the particle diameter, $D>L_{5}{ }^{\prime}$ and no conformational change inside particles was expected and observed, confining DL-OPE $\mathbf{E}_{\mathbf{2 1}}$ in the particle core should lead to significant changes in the label to label distance distribution which might be accessible with the method described above. Therefore, we prepared two particle samples generated from $\mathbf{P E G - O P E _ { 9 }}$ with different $\mathbf{O P E}_{\mathbf{2 1}}$-content, high (a) and low (b). The DEER data (Fig. 4A and B) suggest contributions at distances shorter than $1.5 \mathrm{~nm}$ as well, which are inaccessible by $\mathrm{DEER}^{22}$ but can be detected by spectral linebroadening in continuous-wave ESR. Thus, additional cw-ESR measurements were performed (Fig. 4C).

Both datasets (DEER and cw-ESR) can be fitted independently with a single three dimensional Rice distribution, ${ }^{50}$ yielding each $\mu$, the distance between the mean positions of spatially three dimensional normal distributed spin labels, with a standard deviation of these distributions $\sigma$ ( $c f$. ESI $\dagger$ for details on data analysis). We chose a Rice distribution in this case because no specific model for the distance distribution obtained exists, contrary to the WLC model used for the DL-OPEs in solution. Furthermore, model-free Tikhonov regularization is unreliable due to the signal-to-noise ratio given for this sample. On the contrary, it does not yield more information than the Rice 


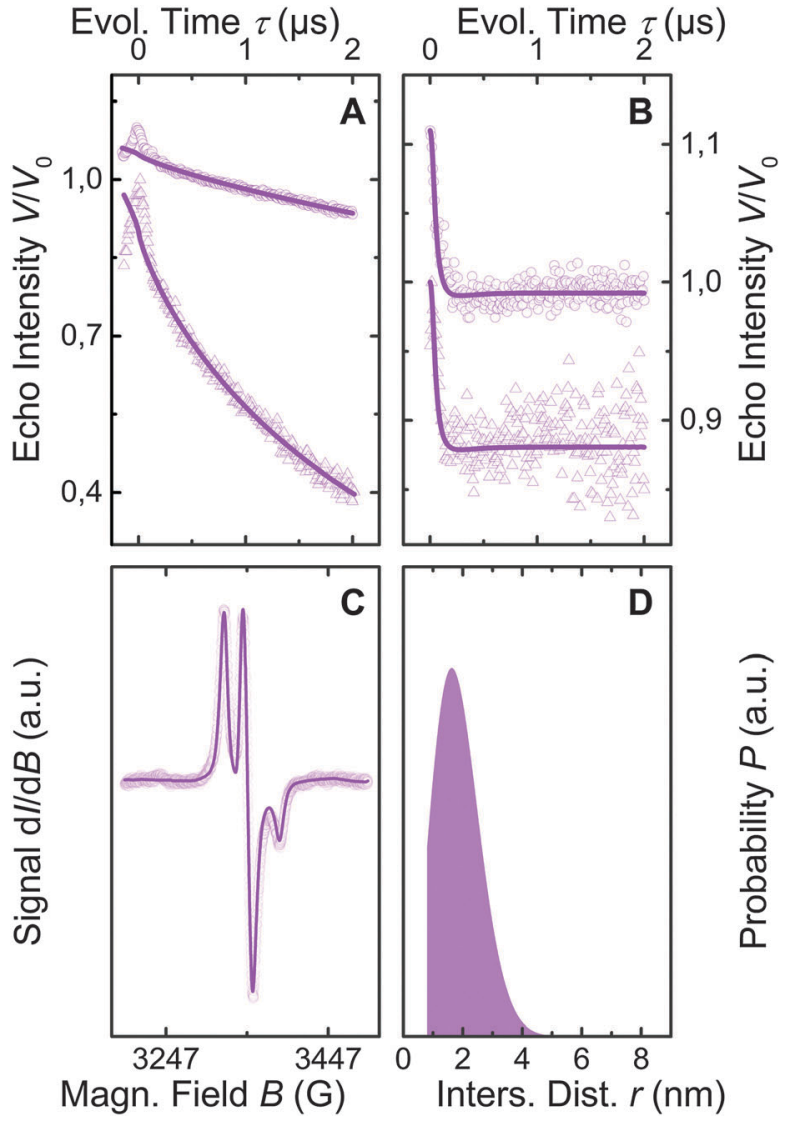

Fig. 4 (A) Normalized DEER traces recorded at $50 \mathrm{~K}$ in Q-band, with intermolecular background contribution of $\mathrm{DL}^{-O P E_{21}}$ in particles with high (triangles) and low (circles) $\mathrm{OPE}_{21}$-content. The slopes of these contributions correspond to the concentrations of these samples (see ESI $\dagger$ ). (B) Corresponding background corrected DEER form factors with a mutual fit of a distance distribution obtained from DEER and CW-ESR. Modulation depths are in the same range as for comparable particle samples with $\mathrm{DL}^{-} \mathrm{OPE}_{5}$ as the probe. Stretched vertically by a factor of 2.5 in the case of low (circles) $\mathrm{OPE}_{\mathbf{2 1}}$-content for better comparison. (C) $\mathrm{CW}$-ESR spectrum of DL-OPE 21 in particles with low $\mathrm{OPE}_{21}$-content recorded at $120 \mathrm{~K}$ in X-band (circles) together with mutual fit of a distance distribution obtained from DEER and cw-ESR (line). (D) The resulting three dimensional Rice interspin distance distribution.

distribution, which is also more stable in the data analysis due to its small number of parameters. Since cw-ESR and DEER cover complementary distance ranges, the parameters obtained by individual fitting of DEER and cw-ESR data do not match exactly, ${ }^{22}$ see Table 2 for all parameters. In order to obtain a mutual fit, these parameters were averaged. The result is shown in Fig. 4D.

Consequently, these measurements prove reproducible, defined spatial arrangement of DL-OPE $\mathbf{E}_{\mathbf{2 1}}$ inside nanoparticles. Possible intermolecular contributions to the observed dipolar interaction are discussed as well (ESI $\dagger$ ) and are negligible.

Taken together, it can be concluded that $\mathbf{D L}-\mathbf{O P E}_{21}$ is incorporated approximately quantitatively and in a defined bent conformation inside nanoparticles. This results in a label to label distance distribution that differs significantly from a DL-OPE $_{21}$ in solution (Fig. 5) leading to the conclusion, that $\mathbf{O P E}_{21}$ bends itself in order to fit into the nanoparticle.
Table 2 Parameters of the individual Rice interspin distance distributions $P(r)$ of DL-OPE $\mathbf{2}_{\mathbf{2 1}}$ in $\mathrm{PEG} \mathrm{POPE}_{\mathbf{9}}-\mathrm{a} / \mathrm{b}$ particles obtained from DEER and $\mathrm{CW}$-ESR. The results as presented in Fig. 4 are based on the average of these parameters

\begin{tabular}{lllll}
\hline Probe DL- & Environment & Method & $\mu^{a}[\mathrm{~nm}]$ & $\sigma^{b}[\mathrm{~nm}]$ \\
\hline OPE $_{21}$ & PEG-OPE $_{9}$-a & DEER & 0 & 1.3 \\
OPE $_{21}$ & PEG-OPE $_{\mathbf{9}}$-b & DEER & 0 & 1.3 \\
OPE $_{\mathbf{2 1}}$ & PEG-OPE $_{\mathbf{9}}$-b & cw-ESR & 0 & 1.0
\end{tabular}

${ }^{a}$ Distance between the mean positions of spatially three dimensional normal distributed spin labels. ${ }^{b}$ Standard deviation of these distributions.

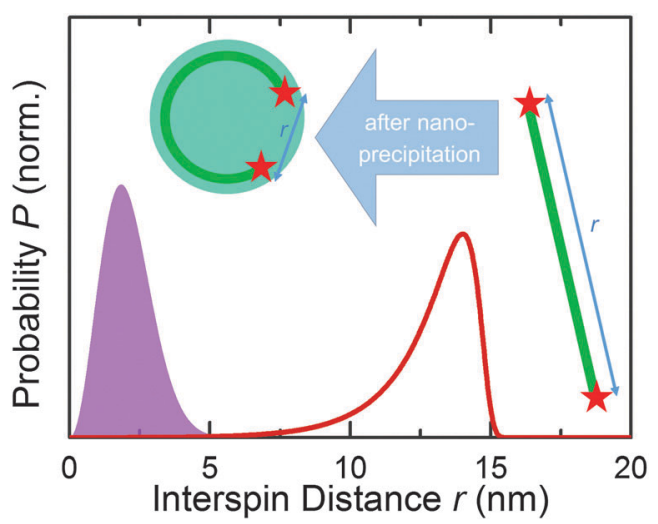

Fig. 5 Comparison of the experimental interspin distance distribution $P(r)$ of $\mathrm{DL}^{-O \mathrm{OPE}_{21}}$ in solution (extrapolated, red line) with the distribution in particles from DEER and cw-ESR data (purple, filled).

Considering defined uniform bending with the average distance between the mean label positions $\mu=0 \mathrm{~nm}$, this leads to a uniform bending diameter $d$ determined by the chain length $L_{21}$ as the circumference of a circle. In this case, we obtain an average bending diameter of $d=4.9 \mathrm{~nm}$. This is in good agreement with the particle size obtained by TEM and DLS, as well as with the calculated optimal particle diameter $D^{*}=6.6 \mathrm{~nm}(\mathrm{ESI} \dagger)$ and the expected core diameter of about the OPE 9 contour length $L_{9}=6.2 \mathrm{~nm}$.

To rationalize these findings, a consideration of the molecular interactions and energetic relationships involved is instructive. Comparing now this bent state of $\mathbf{O P E}_{21}$ in a spherical nanoparticle with an elongated state (Table 3), we are interested in the difference in Gibbs energy between both states.

Bending of such a rigid rod-like oligomer can be considered as the uniform, elastic bending of a cylindrical rod. In this case, the bending energy is given by $H_{\mathrm{B}}=\frac{1}{2} E I \int_{0}^{L} K(p)^{2} \mathrm{~d} p$, with $E$, the Young's Modulus, $I$, the second moment of area, and $K$, the curvature of the rod at point $p$. The product $E I$ can be expressed as the product of persistence length with thermal energy: $E I=$ $L_{p} k T .^{51}$ The persistence length has been determined to $L_{p}=$ $14 \mathrm{~nm}$ at room temperature. ${ }^{14}$ As a simple model one can consider the conformation of $\mathbf{O P E}_{21}$ inside the nanoparticles to be a circle. In this case, the bending energy at room temperature, corresponding to the enthalpy of the bent state, can be derived and is given in Table 3 . 
Table 3 Illustrations of the bent and elongated states with corresponding enthalpies and the difference in entropy between the bent and elongated states

Bent state Elongated state

$$
\text { Chain only }
$$
Ellipsoidal particle

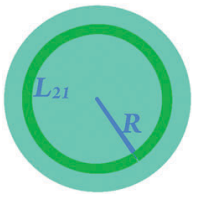 \\ ○
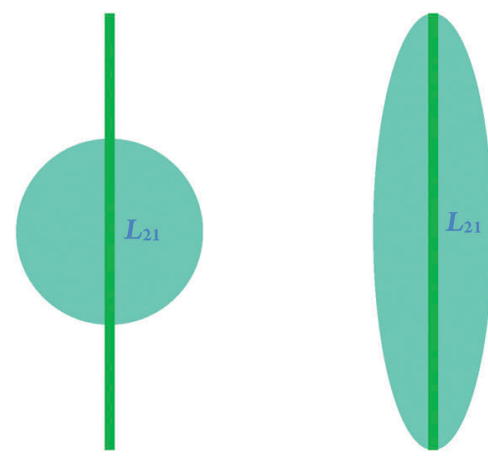 \\ $H_{\mathrm{B}}=\frac{L_{\mathrm{p}} L_{21}}{2 R^{2}} R T$ $=14 \mathrm{~kJ} \mathrm{~mol}^{-1}$ \\ $H_{\mathrm{E}}=500 \mathrm{~kJ} \mathrm{~mol}^{-1}$ \\ $H_{\mathrm{E}}=500 \mathrm{~kJ} \mathrm{~mol}^{-1}$}

from bent to elongated: $T \Delta S \approx+144 \mathrm{~kJ} \mathrm{~mol}^{-1}$

The enthalpy of the elongated state of an $\mathbf{O P E}_{21}$ incorporated into a nanoparticle, where the chain sticks out of the nanoparticle core is dominated by its surface energy. ${ }^{52}$ In a particle core constituted by PEG-OPE $\mathbf{9}_{\mathbf{9}}$, twelve repeat units of $\mathbf{O P E}_{21}$ then would protrude. The surface tension $\gamma$ of the OPEs can be approximated by comparison with similar compounds. For polystyrene it was found ${ }^{53}$ to be $\gamma=40 \mathrm{~mJ} \mathrm{~m}^{-2}$, which agrees with values for several aliphatic hydrocarbons. ${ }^{54}$ Since the OPE's surface should be dominated by the EtHex-sidechains, this should be a satisfying approximation. In this case, the resulting surface energy, corresponding to the enthalpy of the elongated state, is given in Table 3. Elongation of the particle would involve comparable amounts of surface energy, $c f$. ESI $\dagger$ for details.

Restriction of conformational space of the oligomer chain gives rise to entropic cost involved with the incorporation into nanoparticles. The chain can be modelled as a harmonic segmented chain. ${ }^{14}$ One can approximate that about half of the torsions of each segment of the chain are not accessible when $\mathbf{O P E}_{21}$ is confined at a surface, which is the inner perimeter of the particle in this case. The resulting change of entropy is given in Table 3, for details see ESI. $\dagger$

Summarizing the contributions of the bending energy, the surface energy and the entropy, we obtain a difference in the Gibbs energies of both states of $\Delta G \approx 350 \mathrm{~kJ} \mathrm{~mol}^{-1} \approx 140 \mathrm{kT}$ at room temperature. It is mainly dominated by surface energy. This is the reason, why bending is energetically favorable for the $\mathbf{O P E}_{2 \mathbf{1 1}}$. We would like to point out, that the cost of entropy involved with confining the chain in the nanoparticle is about one order of magnitude larger than the bending energy in this case.
It remains unclear at this point, why the observed PEG-OPE $\mathbf{9}_{\mathbf{9}}$ particles are smaller when coprecipitated with $\mathbf{O P E}_{\mathbf{2 1}}$. To this end, a consideration of the expected micelle size of particles formed by PEG-OPE $_{\mathbf{9}}$ is instructive. With the assumption of a densely packed interior and a roughly spherical micelle, the micelle radius $R$ is given by $\frac{4}{3} \pi R^{3}=N \delta a^{2}$, with $\delta$, the mean distance between the polar headgroup and any atom in the apolar part of the surfactant molecule, and $N$, the aggregation number. It can be shown, ${ }^{55}$ that the optimum aggregation number is given by $N^{*}=\frac{49 \pi \gamma \delta^{2}}{48 k T}$.

The average distance between the polar head and any atom in the apolar part within a coil-rod-coil polymer is approximately $\delta=\frac{L_{n}}{4}$ with $L_{n}=(0.69 \mathrm{~nm}) n$ and $n$, the number of repeat units of the $\mathbf{O P E}_{\boldsymbol{n}}$ of interest. Thus, the optimum micelle radius for PEG-OPE $_{9}$ can be expressed as

$$
R^{*}=\frac{L_{9}}{4} \sqrt[3]{\frac{349 a^{2} \gamma}{4}}
$$

With the aforementioned surface tension the micelles are calculated to have an optimum diameter of $D^{*}=6.6 \mathrm{~nm}$, which is close to the $\mathbf{O P E}_{9}$ contour length, $L_{9}=6.1 \mathrm{~nm}$. Since this is less than half of its persistence length $L_{\mathrm{p}}$, the rigid $\mathbf{O P E}_{\mathbf{9}}$ cannot arrange freely inside the particle core. Thus, the implicit assumption of a densely packed interior does not apply. In this case, incorporating $\mathbf{O P E}_{21}$ into the core of a

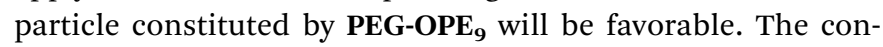
tour length of $\mathbf{O P E}_{21}$ is approximately equal to the persistence length, $L_{21} \approx L_{\mathrm{p}}$, allowing a densely packed interior of the particle via chain bending.

\section{Conclusions}

To date, the individual conformation of rod-like conjugated polymer chains inside nanoparticles was an open question, especially within particles that are smaller in size than the contour length of the 'rigid' chain. In summary, we find, that particles from amphiphilic coil-rod-coil block copolymers with short chained $\left(L_{n}<L_{\mathrm{p}}\right)$ rod blocks decrease in size when mixed with long chain derivatives $\left(L_{n} \approx L_{\mathrm{p}}\right)$ prior to nanoprecipitation, providing access to nanoparticles $D<10 \mathrm{~nm}$. Chain bending of the long chains enables efficient packing and an energetically more favorable particle size is obtained. ESR spectroscopy provides a unique way of characterizing the conformation of individual polymer chains inside nanoparticles.

\section{Acknowledgements}

We thank G. Jeschke and C. Peter for fruitful discussion and valuable input. Financial support by the DFG (Me1388/7-1, DR 743/7-1) is gratefully acknowledged. S. M. was supported 
through the University of Konstanz by the 'Free space of creativity' program within the federal excellence initiative.

\section{References}

1 J. H. Burroughes, D. D. C. Bradley, A. R. Brown, R. N. Marks, K. Mackay, R. H. Friend, P. L. Burns and A. B. Holmes, Nature, 1990, 347, 539-541.

2 M. C. Scharber, D. Mühlbacher, M. Koppe, P. Denk, C. Waldauf, A. J. Heeger and C. J. Brabec, Adv. Mater., 2006, 18, 789-794.

3 S. Günes, H. Neugebauer and N. S. Sariciftci, Chem. Rev., 2007, 107, 1324-1338.

4 J. Chen and Y. Cao, Acc. Chem. Res., 2009, 42, 1709-1718.

5 J. Peet, A. J. Heeger and G. C. Bazan, Acc. Chem. Res., 2009, 42, 1700-1708.

6 A. Mishra, C.-Q. Ma and P. Bäuerle, Chem. Rev., 2009, 109, 1141-1276.

7 Y.-J. Cheng, S.-H. Yang and C.-S. Hsu, Chem. Rev., 2009, 109, 5868-5923.

8 C. J. Brabec, V. Dyakonov and U. Scherf, Organic photovoltaics: materials, device physics, and manufacturing technologies, Wiley-VCH, Weinheim, 2008.

9 S. W. Thomas, G. D. Joly and T. M. Swager, Chem. Rev., 2007, 107, 1339-1386.

10 A. C. Grimsdale, K. Leok Chan, R. E. Martin, P. G. Jokisz and A. B. Holmes, Chem. Rev., 2009, 109, 897-1091.

11 H. Hoppe and N. S. Sariciftci, in Photoresponsive Polymers II, ed. S. R. Marder and K.-S. Lee, Springer, Berlin Heidelberg, 2007, pp. 1-86.

12 C. Wu and D. T. Chiu, Angew. Chem., Int. Ed., 2013, 52, 3086-3109.

13 J. Pecher and S. Mecking, Chem. Rev., 2010, 110, 6260-6279.

14 G. Jeschke, M. Sajid, M. Schulte, N. Ramezanian, A. Volkov, H. Zimmermann and A. Godt, J. Am. Chem. Soc., 2010, 132, 10107-10117.

15 G. Jeschke, Annu. Rev. Phys. Chem., 2012, 63, 419-446.

16 A. D. Milov, K. M. Salikhov and M. D. Shirov, Phys. Solid State, 1981, 23, 565-569.

17 Y. D. Tsvetkov, A. D. Milov and A. G. Maryasov, Russ. Chem. Rev., 2008, 77, 487.

18 G. Jeschke, ChemPhysChem, 2002, 3, 927-932.

19 A. Milov, A. Ponomarev and Y. Tsvetkov, Chem. Phys. Lett., 1984, 110, 67-72.

20 G. Jeschke, Macromol. Rapid Commun., 2002, 23, 227-246.

21 R. Ward, A. Bowman, E. Sozudogru, H. El-Mkami, T. OwenHughes and D. G. Norman, J. Magn. Reson., 2010, 207, 164-167.

22 J. E. Banham, C. M. Baker, S. Ceola, I. J. Day, G. H. Grant, E. J. J. Groenen, C. T. Rodgers, G. Jeschke and C. R. Timmel, J. Magn. Reson., 2008, 191, 202-218.

23 G. Jeschke and Y. Polyhach, Phys. Chem. Chem. Phys., 2007, 9, 1895-1910.

24 S. Pornsuwan, G. Bird, C. E. Schafmeister and S. Saxena, J. Am. Chem. Soc., 2006, 128, 3876-3877.
25 B. E. Bode, D. Margraf, J. Plackmeyer, G. Dürner, T. F. Prisner and O. Schiemann, J. Am. Chem. Soc., 2007, 129, 6736-6745.

26 J. E. Lovett, M. Hoffmann, A. Cnossen, A. T. J. Shutter, H. J. Hogben, J. E. Warren, S. I. Pascu, C. W. M. Kay, C. R. Timmel and H. L. Anderson, J. Am. Chem. Soc., 2009, 131, 13852-13859.

27 A. Godt, M. Schulte, H. Zimmermann and G. Jeschke, Angew. Chem., Int. Ed., 2006, 45, 7560-7564.

28 G. I. Likhtenshtein, J. Yamauchi, S. Nakatsuji, A. I. Smirnov and R. Tamura, Nitroxides: Applications in Chemistry, Biomedicine, and Materials Science, Wiley-VCH Verlag GmbH \& Co. KGaA, Weinheim, Germany, 2008.

29 J. P. Klare and H.-J. Steinhoff, Photosynth. Res., 2009, 102, 377-390.

30 W. L. Hubbell, H. S. Mchaourab, C. Altenbach and M. A. Lietzow, Structure London, England: 1993, 1996, vol. 4, pp. 779-783.

31 A. P. Todd, J. Cong, F. Levinthal, C. Levinthal and W. L. Hubbell, Proteins, 1989, 6, 294-305.

32 C. Altenbach, T. Marti, H. G. Khorana and W. L. Hubbell, Science, 1990, 248, 1088-1092.

33 U. H. F. Bunz, Macromol. Rapid Commun., 2009, 30, 772-805.

34 C. Weder, Poly(arylene etynylene)s, Advances in Polymer Science, Springer Berlin Heidelberg, Berlin, Heidelberg, 2005, vol. 177.

35 Y. Shirai, A. J. Osgood, Y. Zhao, K. F. Kelly and J. M. Tour, Nano Lett., 2005, 5, 2330-2334.

36 W. R. Browne and B. L. Feringa, Nat. Nanotechnol., 2006, 1, 25-35.

37 S. Höger, J. Weber, A. Leppert and V. Enkelmann, Beilstein J. Org. Chem., 2008, 4, 1-15.

38 J. S. Moore and J. Zhang, Angew. Chem., Int. Ed., 1992, 31, 922-924.

39 Q. Lu, K. Liu, H. Zhang, Z. Du, X. Wang and F. Wang, ACS Nano, 2009, 3, 3861-3868.

40 B. Albinsson and J. Mårtensson, J. Photochem. Photobiol., C, 2008, 9, 138-155.

41 J. Rochford and E. Galoppini, Langmuir, 2008, 24, 5366-5374.

42 P. F. H. Schwab, M. D. Levin and J. Michl, Chem. Rev., 1999, 99, 1863-1934.

43 F. Schütze, M. Krumova and S. Mecking, Macromolecules, 2015, 48, 3900-3906.

44 C. Wu, C. Szymanski and J. McNeill, Langmuir, 2006, 22, 2956-2960.

45 C. Wu, B. Bull, C. Szymanski, K. Christensen and J. McNeill, ACS Nano, 2008, 2, 2415-2423.

46 C. S. Fischer, M. C. Baier and S. Mecking, J. Am. Chem. Soc., 2013, 135, 1148-1154.

47 C. Szymanski, C. Wu, J. Hooper, M. A. Salazar, A. Perdomo, A. Dukes and J. McNeill, J. Phys. Chem. B, 2005, 109, 8543-8546.

48 F. Schütze, B. Stempfle, C. Jüngst, D. Wöll, A. Zumbusch and S. Mecking, Chem. Commun., 2012, 48, 2104-2106.

49 G. Jeschke, V. Chechik, P. Ionita, A. Godt, H. Zimmermann, J. Banham, C. R. Timmel, D. Hilger and H. Jung, Appl. Magn. Reson., 2006, 30, 473-498. 
50 S. Domingo Köhler, M. Spitzbarth, K. Diederichs, T. E. Exner and M. Drescher, J. Magn. Reson., 2011, 208, 167-170.

51 M. R. K. Mofrad and R. D. Kamm, Cytoskeletal mechanics: models and measurements, Cambridge University Press, Cambridge, 2006.

52 D. Chandler, Nature, 2005, 437, 640-647.
53 I. T. S. Li and G. C. Walker, J. Am. Chem. Soc., 2010, 132, 6530-6540.

54 L. A. Girifalco and R. J. Good, J. Phys. Chem., 1957, 61, 904-909.

55 L. Maibaum, A. R. Dinner and D. Chandler, J. Phys. Chem. B, 2004, 108, 6778-6781. 